\title{
Infant Screening System Based on Cry Analysis
}

\author{
Akshay Mohan Mendhakar ${ }^{*}$, Sreedevi. ${ }^{2}$, Arunraj K ${ }^{3}$, Jayashree Shanbal ${ }^{4}$ \\ ${ }^{1}$ DHLS Mysuru Center, All India Institute of Speech \& Hearing, INDIA \\ ${ }^{2}$ Department of Clinical services, All India Institute of Speech \& Hearing, INDIA \\ ${ }^{3}$ Department of Audiology, All India Institute of Speech \& Hearing, INDIA \\ ${ }^{4}$ Department of Speech Pathology, All India Institute of Speech \& Hearing, INDIA \\ * Corresponding author email: amendhakar@gmail.com
}

Received: 05 July 2018 / Accepted: 23 September 2018 / Published: 02 October 2018

\begin{abstract}
Acoustical investigation of infant cries has been a clinical and research focus in the recent years. Findings of several studies reveal the importance of cry as a useful window for early detection of several diseases and communication difficulties such as hearing impairment, intellectual disabilities, cerebral palsy etc. This motivates us to use a minimal interface system that can automatically classify infant cries into normal and pathological with the help of state-of-the-art machine learning strategies. In this paper, we propose a software program for screening infants based on their cries. The proposed system is able to detect \& classify infant cries into normal and pathological based on the acoustic input. To build and train the system, infant cries of normal and Low Birth Weight (LBW) newborn within 7 days of birth were considered. A pain induced cry elicited using the routine intramuscular immunization was recorded using a standard Olympus LS-100 recorder which was held about 10 centimetres away from the infant's mouth. The acoustic correlates of these cries were used to build the software tool. Artificial Neural Network was employed to improve its functionality. Therefore, we propose a screening tool for further accessibility and large-scale implementation.

Keyword: Acoustic, Analysis, Cry, Early intervention, Infant, Screening.
\end{abstract}

\section{Introduction}

Infant cry is the actual means of communication of the baby in the first month of life that raises questions of interpretation in the adult parent/caretaker. Cry is the combination of the functions of brain, the larynx and oral cavity. Cry as a social behaviour, has powerful effects on the parent-child relationship that elicits strong emotions in parents. Infant cry analysis for clinical purposes has been gearing up with its importance in the area of communication disorders in terms of early identification and prevention of communication disorders. Studies on acoustic analysis of infant cries in the clinical population such as hearing impairment has revealed significant difference in spectral parameters such as fundamental frequency (F0) and the dominant frequency in infants [1]. In other conditions such as autism, deviancies in spectral parameters such as $\mathrm{F} 0$ were observed and many have opined that this kind of an atypical vocal signature could lead to early identification of the conditions [2]. As discussed in the reports of [3], no difference in the cry characteristics of LBW infants compared to full term infants with normal birth weight. Several studies have investigated developmental outcome for preterm children and showed a greater incidence of intellectual functioning deficits in early and middle childhood [4] as well as increased occurrence of motor dysfunction, learning disabilities, behavioral disorders, difficulties in language comprehension and expression and phonological development [5]-[8].

Three possible explanations for poor speech and language outcomes in preterm infants have been proposed [9]. The first one attributes poor outcome to overall poorer intellectual development. The second possibility is that the 
preterm infant's eventual speech and language behavior is a result of poorer environmental support, with a tendency for premature birth to be associated with lower socioeconomic status. The third possibility states the neurophysiological disturbances arising from complications of premature birth as the reason for the presence of speech language difficulties in them. This would suggest that preterm infants demonstrate a neurophysiological disorder at birth, which may have a detrimental impact on their eventual communicative behavior. Assuming an infant's vocal output is closely regulated by the child's neurophysiology [10]-[11], it is conceivable that abnormalities may be present in the earliest of vocal behaviors i.e. infant cry.

Cry results of the premature infants differed to that of normal healthy full term infants and the studies have revealed that more the premature infant, higher the fundamental frequency, shorter the duration, and more of high pitched [12]. Long time average spectrum (LTAS) of 10 full term and 10 preterm infants showed significant difference in parameters such as higher first spectral peak (FSP) values in preterm babies which indicated a higher vocal F0 [13]. However, similar attempt showed contrast finding of lower mean FSP in infants who were born preterm than of full term infants which shows lower fundamental frequency in them [14]. If crying behavior relies on the same anatomical systems that regulate speech, then identification of abnormalities in the crying of preterm infants may provide potentially useful information related to speech outcome. In the Indian arena, previous work has shown a significant variation in acoustic characteristics of cries of infants with neurological damage, prematurity, medical conditions, SIDS and many other risk factors. Various studies have indicated that the most of the acoustic parameters including frequency related, intensity related parameters can differentiate normal and abnormal cries of newborns [15].

Earlier study by the current authors was one among the pilot studies which aimed to build a screening tool based on acoustic parameters of infant cry [16]. Even though it was a commendable effort the effectiveness of the tool was limited with poor sensitivity and specificity.
Therefore, the present study planned to verify the effectiveness of the acoustic parameters highlighted across the literature and work on the shortcomings of our previous study by utilising machine learning algorithms. Thus, the specific objectives of the current study were to compare acoustic characteristics of infant cries in wellborn nursery babies and babies with preterm low birth weight and implement ANN into the screening tool to improvise its effectiveness.

\section{Materials and Methods}

The study was carried out in two phases. The first phase involved investigating acoustic parameters of cry. The second phase involved building an ANN to improve the effectiveness of the screening tool.

\subsection{Phase I: Acoustic characteristic extraction}

\subsubsection{Participants}

A total of 30 newborns with equal number of wellborn nursery and preterm LBW newborns within seven days of birth were considered for this study. All infants were born at the government hospital located in and across Mysuru city in the state of Karnataka. The wellborn babies fulfilled the screening criteria, as the babies were full term healthy with no other pre/peri/post complications as observed and reported in the medical case file. Newborns with preterm low birth weight include babies with birth weight of $<1800$ gram and a gestational age of less than 37 weeks at birth. All the preterm LBW infants received intensive care at the neonatal intensive care unit (NICU). The primary diagnoses for infants were low birth weight with prematurity.

\subsubsection{Procedure}

The pain induced cries in the wellborn babies were recorded after it received a routine intramuscular immunization (Hepatitis B). During this procedure none of the infants received any kind of behavioural and/or pharmacological support to control their pain. Since vaccination was not provided to the preterm low birth weight babies, alternate pain evoking method was used as tapping on the nose 
Mendhakar et al., Int. Ann. Sci.; Vol. 6, Issue 1, pp: 1-7, 2019

of the infant. The researcher carried out cry recording while the nursing staff performed the immunization procedure for wellborn nursery babies. The audio recorder Olympus LS-100 recorder $(48 \mathrm{kS} / \mathrm{s})$ was positioned in front of the newborns with an external microphone situated $10 \mathrm{cms}$ away from the infant's mouth. The recorded infant cries in WAV format were transferred to a PC and converted into .wav files for acoustic analyses. Demographic details of the infants were also obtained.

\subsubsection{Acoustic Analyses}

PRAAT software version 5.4.01[17] was used for the acoustic analysis. Analysis for the initial 15 seconds of each cry sample, which was selected and segmented from the total cry samples were carried out to extract the acoustics parameters. Frequency, Intensity and Formant parameters were extracted from those samples from the wide band spectrographic displays as shown in Figure 1,2 and 3 .

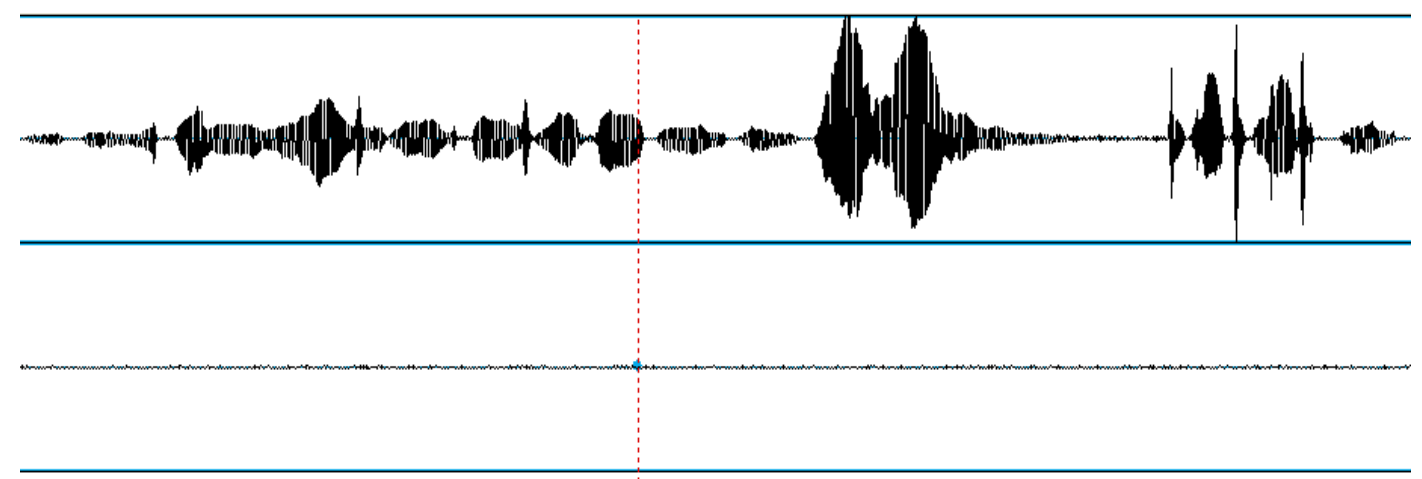

Figure 1: wave form representation of total cry sample
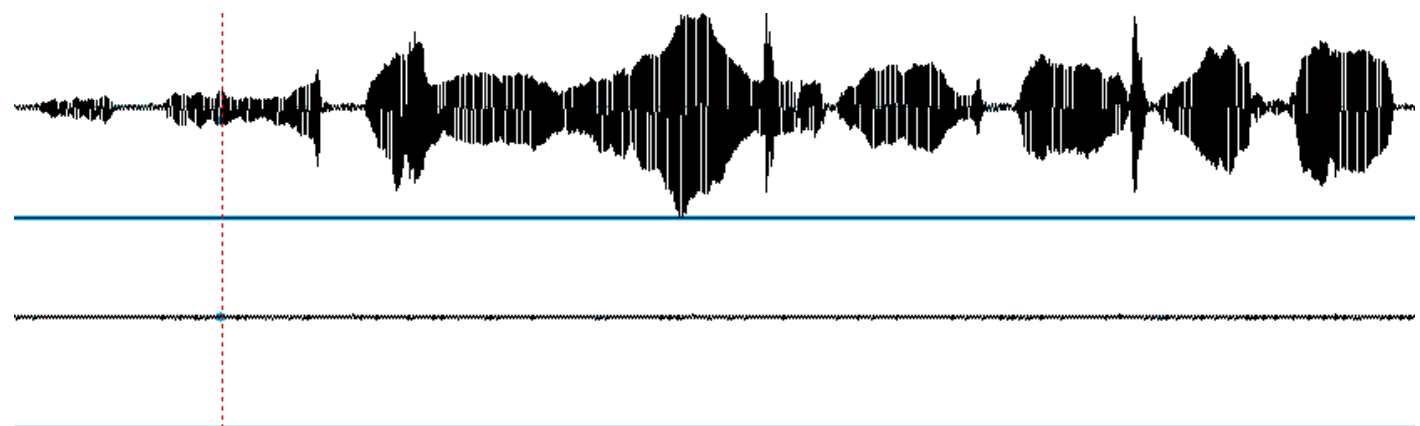

Figure 2: Wave form representation of selected $15 \mathrm{sec}$ cry duration from the onset
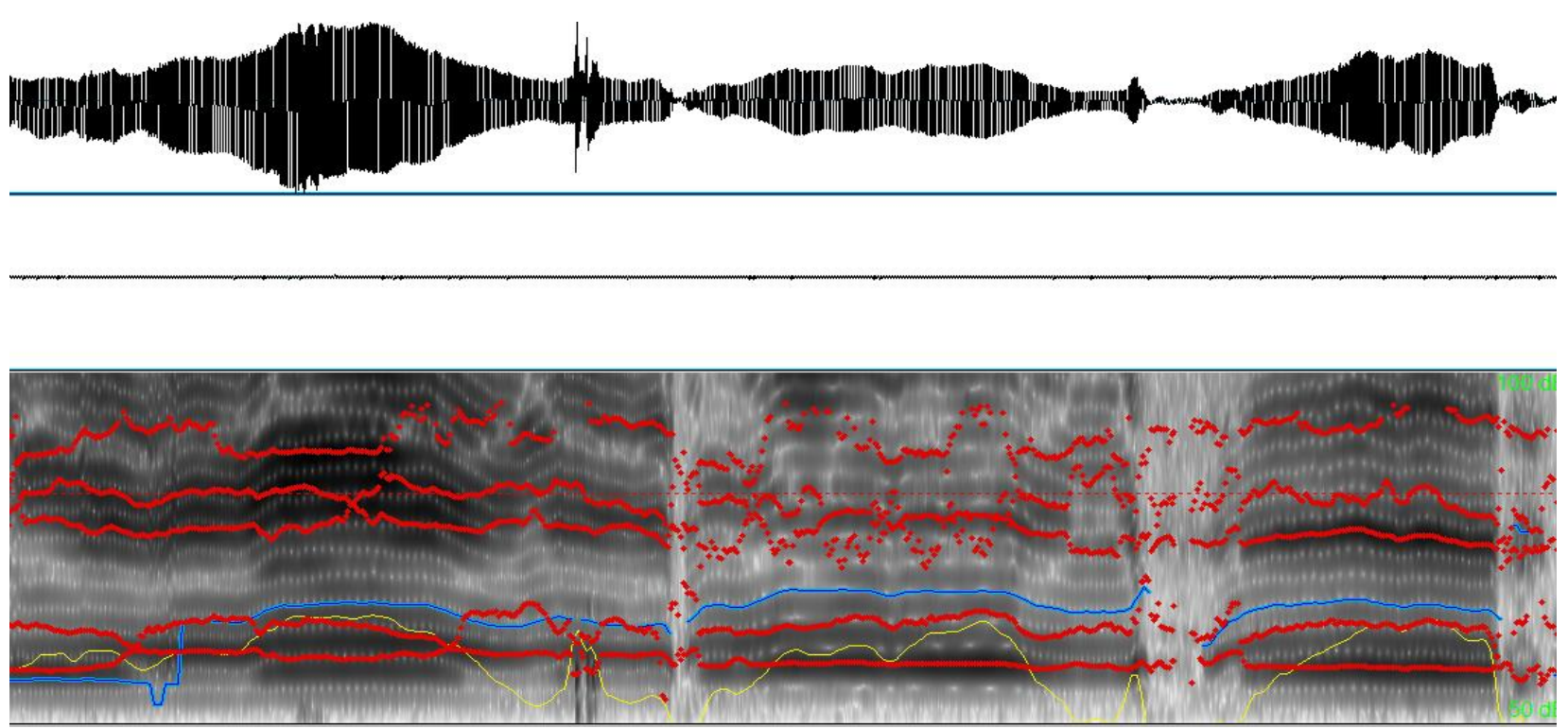

Figure 3: Spectrogram representation of the cry. Formants (Red lines), Intensity (Yellow) and Pitch contour (Blue lines) are shown. 
Mean and standard deviation (SD) values for each parameter in preterm LBW and well born nursery babies were tabulated in SPSS- Statistical Package for Social Sciences ver 17.0. If $p$ value was below 0.05 it was considered to be statistically significant as the data did not achieve normality, Mann- Whitney U-statistic was employed to evaluate the differences between preterm LBW infants and wellborn nurseries.

\subsection{Phase II: Building ANN Based Screening Tool}

Based on the sensitivity analysis key input variables found to be important in differentiating normal vs LBW cries were considered for building the prediction model. The multilayer ANN used in the current study was a feedforward network with sigmoid activation functions in the hidden layers and a linear activation function in the output node in MATLAB ver 2015a. The ANN employed in this study was trained using back propagation algorithm with momentum terms and gradient descent. Optimal number error approach was used to specify the maximum number of training epochs, learning rates, number of nodes in a hidden layer. During training phase of the ANN, parameters such as number of nodes were varied between 6 to 24 with various learning rates in increments of 0.05 from 0.01 to 1.0. Based on the variable of Mean Square Error (MSE) variable among the measured data and the model output the ANN was modelled. Randomization of the model weights was done during the training process and was terminated when over fitting of the data was seen.

\section{Results}

\subsection{Phase I: Acoustic Characteristics Extraction}

The current study aimed to investigate the acoustic differences in cries of wellborn nursery babies and in babies with preterm low birth weight (LBW). The results are compared and discussed separately under fundamental frequency, formants, number of pulses and degree of voice breaks. Table I reveals the mean, standard deviation (SD) and range of cry variables obtained for both preterm LBW and wellborn nursery babies. The present study revealed a number of significant differences in the cry characteristic in both the groups.

\subsubsection{Fundamental Frequency (F0)}

Table 1 displays the mean and standard deviation of average, minimum, maximum and median fundamental frequency of all the 30 infants. As observed, the results indicated slightly lower values of average, minimum and maximum fundamental frequencies in preterm LBW babies compared to well born babies. However, Mann Whitney U statistic revealed significant difference $(p=0.008)$ between preterm LBW and well born babies, only for maximum frequency.

Table 1: The mean, SD and range of cry variables obtained in preterm $L B W$ and wellborn babies

\begin{tabular}{|l|l|l|l|l|l|}
\hline \multirow{2}{*}{$\begin{array}{l}\text { Acoustic } \\
\text { Parameters }\end{array}$} & \multicolumn{2}{|c|}{ Normal infants } & \multicolumn{2}{c|}{ Low birth weight infants } & \multirow{2}{*}{$\boldsymbol{p}$ - value } \\
\cline { 2 - 5 } & Mean (SD) & Range & Mean (SD) & \\
\hline Average F0 & $491(44)$ & $409-553$ & $461(67)$ & $355-562$ & 0.206 \\
\hline Minimum F0 & $185(77)$ & $76-350$ & $175(84)$ & $77-348$ & 0.760 \\
\hline Maximum F0 & $1151(89)$ & $959-1255$ & $870(267)$ & $492-119$ & $0.008^{*}$ \\
\hline Median F0 & $486(40)$ & $404-546$ & $449(59)$ & $353-542$ & 0.089 \\
\hline Mean intensity & $63(4)$ & $58-72$ & $62(4)$ & $56-70$ & 0.337 \\
\hline No. of pulses & $4045(755)$ & $2748-5767$ & $3373(869)$ & $2962-2068$ & $0.050^{*}$ \\
\hline No. Voice breaks & $24(6)$ & $2-35$ & $26(8)$ & $16-41$ & 0.336 \\
\hline Degree of voice break & $37(12)$ & $17-56$ & $49(11)$ & $34-63$ & $0.050^{*}$ \\
\hline F1 & $1155(94)$ & $1000-1291$ & $1076(73)$ & $938-1192$ & $0.029^{*}$ \\
\hline F2 & $2182(172)$ & $1980-2601$ & $1921(133)$ & $1596-2111$ & $0.000^{*}$ \\
\hline F3 & $3274(102)$ & $3087-3458$ & $3189(110)$ & $3328-3189$ & 0.089 \\
\hline F4 & $4192(212)$ & $779-3887$ & $4202(89)$ & $4046-4357$ & 0.116 \\
\hline
\end{tabular}




\subsubsection{Formants}

Analysis on formants indicated lower F1, F2 and $\mathrm{F} 3$ and higher $\mathrm{F} 4$ values in preterm LBW infants when compared to the control group. Statistical evaluation revealed significantly different $\mathrm{F} 1$ $(\mathrm{p}=0.029)$ and F2 $(\mathrm{p}=0.00)$. No significant difference was noticed in the F3 $(\mathrm{p}=0.089)$ and F4 $(p=0.116)$ values.

\subsubsection{No of pulses and Degree of voice breaks}

Preterm LBW infants showed decrease in $(p=0.05)$ number of pulses and increase in degree of voice breaks $(p=0.05)$ significantly compared to well born infants. Number of pulses in the signal represents the voiced component of the cry signal. Lower number of pulses in the cry signal of preterm LBW infants indicates less voiced sounds in the cry compared to the control group. Similarly, higher degree of voice breaks in preterm LBW infants cry indicate additional silence and unvoiced component in the signal compared to wellborn nursery infants. Subtle differences were noted in intensity and number of voices breaks but statistical significance was not achieved for these parameters.

\subsection{Phase II: Building ANN based screening tool}

An interactive ANN was built as the result of the phase I of this study. By integrating the developed ANN into the previous research tool [16]. We expect to note greater sensitivity and specificity of the proposed tool. Large scale implementation of the improvised tool is necessary to arrive at any conclusive evidence.

\section{Discussion}

The current study evaluated the acoustic characteristics of infant cries in preterm LBW and wellborn nursery infants. The results of the present study revealed significant difference in maximum F0, number of pulses, degree of voice breaks, formant (F1 \& F2). The average maximum $\mathrm{F} 0$ is $870 \pm 267 \mathrm{~Hz}$ for preterm LBW infants which are much lower than that of wellborn nursery infants. Average, minimum and median F0 values also showed lower values in preterm LBW infants but did not show any significant from the control group. Similarly, F1 and F2 values were also lower in preterm LBW babies compared to wellborn nursery infants. However, these results are not in agreement with the earlier studies. Studies on acoustic cry analysis of infants indicated higher minimum fundamental frequency in preterm LBW infants and higher formant values than normal infants [18]. Studies on LTAS measurement in preterm infants demonstrated a high overall first spectral peak across an entire cry sample than their controlled match, which indicates higher vocal F0 in the preterm group [13]. Similar findings of higher F0 in preterm newborns were also reported by [19]. The findings of the earlier studies were attributed to two possibilities. Higher F0 in the cries of preterm infants may simply be related to smaller vocal folds, resulting from physical size differences at birth. Another possibility for the high $\mathrm{F} 0$ in preterm infants could be due to the elicitation of additional stress response for the pain stimuli. During the stress of pain, the laryngeal musculature is tightened, which might have effects of raising F0 [20]-[21]. In contradiction to these justifications [22] suggest that elevated $\mathrm{F} 0$ of spontaneous cries in preterm babies might not be due to their smaller body size but a much more complex neurophysiological state owing to different intra and extra uterine experiences. However, the lower maximum F0 in our study could be attributed to other reasons such as fatigue and exhaustion of the babies due to their continuous crying in the NICU and hence their baseline arousal level could not be controlled in this study. This continuous cry of the preterm LBW infants might lead to fatigue and also the intensity of the cry signal was observed to be reduced in the preterm LBW infants which could possibly result in lower fundamental frequencies. As reported by [23] on preterm infants using LTAS are in support with the current study results. Authors have obtained lower fundamental frequency in preterm babies compared to the full term babies and results were justified based on the external factors such as presence of back ground noise during recording, interaction between the infant and caretaker. The present study also revealed 
decrease in number of pulses in preterm LBW infants indicating reduced voiced sounds in their cry bouts compared to the wellborn nursery infants. The outcome of the analysis also indicated that preterm LBW infants had lesser amount of voicing and or phonation in their total cry duration. This is in consonance with the earlier studies, which indicated lesser amount of phonation and duration of cries produced by preterm infants [12], [24]-[25] [13] measured the percent phonation value i.e. the amount of phonation that elimination of unvoiced segments of cry signal. The result indicated lower percent phonation in preterm babies in the cry signal [13]. Even, the present study also revealed similar results with reduced number of pulses or lesser amount of phonation in preterm LBW compared to wellborn nursery infants. Preterm LBW infants were frequently exposed to intravenous injection as a part of their routine care in NICU. Associated pain, discomfort or distress could have made the infant fatigue, and which would have caused less sub glottal pressure that interrupted the continuous vocal fold vibrations. This could have attributed to the reduced voiced component in cry which was represented as decreased number of pulses in them.

Degree of voice breaks (DVB) was another parameter, which showed significant difference indicating higher value for preterm LBW infants than wellborn nursery infants. DVB is the ratio of total length of voice break areas in the complete sample. Higher degree of voice breaks indicates more of silence and distribution of unvoiced component in the infant cry. Higher degree of voice breaks in preterm LBW infants have been noted in this study. Various authors also supported the findings of the current study indicating higher degree of voice break in highrisk babies [26]. Reports of acoustic parameters [15] using MDVP on normal and high-risk babies showed higher mean value (44.7) of DVB for premature babies compared to normal (30.16) infants. Increased amount of voiceless segments in healthy premature cries in comparison to full term healthy cries was reported by [26]. Authors speculate this is not dependent on gestational age, but on the pathology and the difference in voice breaks were attributed to the immature innervations of the larynx in preterm newborns.

ANN has always been accredited as a black box model, where we cannot precisely determine the relationship between the variables itself. But for an ANN to be effective application of sensitivity analysis is paramount. This could help us associate the relationship between the input acoustic variables and the output classes. A robust ANN should be balanced between its variables of number of layers, the learning rates, number of epochs etc. In this study we used a trial-and error approach to determine the above variables effectively and hence make the ANN robust to classify infant cries.

\section{Conclusion}

In precise, the current study indicated deviancies of acoustic variables in preterm LBW infants compared to full term normal babies in terms of fundamental frequency, formants, voiced components and the degree of voice breaks. The collective results suggested the acoustic parameters that could differentiate the specific high-risk population at an early stage for effective management of such infants and it does offer an acoustic profile of the crying behaviour of preterm LBW infants. Early evaluation of cry and its behaviours may assist us in predicting the developmental outcome of infants at risk for communication disorders.

\section{Declaration}

\subsection{Acknowledgments}

We would like to thank Dr. S R Savithri, Director, AIISH, Mysuru for permitting us to carry out this research study and for the ARF funding. Special thanks to the research officer, Neetu Thoduvayil and the staff of Cheluvamba Hospital, Mysuru, for the providing necessary support.

\subsection{Ethical Approval}

Ethical approval for this study was obtained from All India Institute of Speech and Hearing Authority.

\subsection{Informed Consent}

An informed consent was obtained from all the parents of the infants. 
Mendhakar et al., Int. Ann. Sci.; Vol. 6, Issue 1, pp: 1-7, 2019

\subsection{Conflict of Interest}

The authors declare that they have no conflict of interest.

\section{How to Cite this Article:}

A. Mendhakar, S. N, A. K, and J. Shanbal, "Infant Screening System Based on Cry Analysis", Int. Ann. Sci., vol. 6, no. 1, pp. 1-7, Oct. 2018. Doi: 10.21467/ias.6.1.1-7

\section{References}

[1] G. Várallyay Jr, "Infant cry analyzer system for hearing disorder detection", Transactions on Automatic Control and Computer Science, vol. 49, no.63, pp.57-60, 2004.

[2] G. Esposito and P .Venuti, "Understanding early communication signals in autism: a study of the perception of infant's cry", Journal of Intellectual Disability Research, vol.54, no.3, pp.216-223, 2010.

[3] K. Michelsson, "CRY ANALYSES OF SYMPTOMLESS LOW BIRTH WEIGHT NEONATES AND OF ASPHYXIATED NEWBORN INFANTS", Acta Paediatrica, vol. 60, no. 216, pp. 9-45, 1971.

[4] D. Caputo, K. Goldstein, and H. Taub, "Neonatal compromise and later psychosocial development: A 10year longitudinal study,", S. Friedman \& M. Sigman (Eds.), Preterm birth and psychosocial development, New York: Academic Press, pp. 353-386, 1981.

[5] W. Hunt, Tooley, and D. Harvin ,"Learning disabilities in children with birth weights $<1500$ grams", Seminars in Perinatology, vol.6, 280-287, 1982.

[6] B. Byers-Brown, M. Bendersky,and T. Chapman, "The early utterances of preterm infants", British Journal of Disorders of Communication, vol.21,pp. 307-319,1986.

[7] R. Grunau, S. Kearney and M. Whitfield, "Language development at 3 years in pre-term children of birth weight below $1000 \mathrm{~g}$ ", International Journal of Language \& Communication Disorders, vol. 25, no. 2, pp. 173-182, 1990

[8] D. Aram, M. Hack, S. Hawkins, B. Weissman, and E. Borawski-Clark, "Very-low-birthweight children and speech and language development", Journal of Speech and Hearing Research, vol.34, pp. 1169-1179, 1991.

[9] J. Briscoe, S. Gathercole and N. Marlow, "Short-Term Memory and Language Outcomes After Extreme Prematurity at Birth", Journal of Speech Language and Hearing Research, vol. 41, no. 3, p. 654, 1998.

[10] M. Corwin, B. Lester and H. Golub, "The infant cry: What can it tell us?", Current Problems in Pediatrics, vol. 26, no. 9, pp. 313-334, 1996.

[11] B. Lester, "Cry analysis in preterm and term infants related to developmental outcome at 18 months and five years", Infant Behavior and Development, vol. 9, p. 224, 1986.

[12] K. Michelsson, P. Sirviö, M. Koivisto, A. Sovijärvi and O. Wasz-Höckert, "Spectrographic Analysis of Pain Cry in Neonates with Cleft Palate", Neonatology, vol. 26, no. 5-6, pp. 353-358, 1975.

[13] A. Goberman and M. Robb, "Acoustic Examination of Preterm and Full-Term Infant Cries", Journal of Speech Language and Hearing Research, vol. 42, no. 4, p. 850, 1999.

[14] R. Barr, S. Chen, B. Hopkins and T. Westra, "CRYING PATTERNS IN PRETERM INFANTS", Developmental Medicine \& Child Neurology, vol. 38, no. 4, pp. 345-355, 2008.
[15] N. Sangeetha, "Acoustic analysis of infant cry", Masters in Speech \& Hearing, University of Mysore, 1999.

[16] N. Sreedevi, C. Jayashree Shanbal and K. Arunraj, "Development and Validation of a Computerized Screening Tool for Infant Cry", DEPARTMENTAL PROJECT-DP 119, All India Institute of Speech and Hearing, 2014.

[17] P. Boersma and D. Weenink, "Praat (Version 5.4)[Computer software]", Amsterdam: Institute of Phonetic Sciences., 2006.

[18] L.Rautava ,S. Ojala, R. Parkkola,H. Rikalainen,H. Lapinleimu, L. Haataja, and L. Lehtonen, "Acoustic quality of cry in very low birth weight infants at the age of $1 \frac{1}{2}$ years", Early Human Development, vol.83, pp. 512, 2007.

[19] C. Johnston, B. Stevens, K. Craig, and R. Grunau, "Developmental changes in pain expression in premature, full-term, two- and four-month-old infants", Pain, vol. 52, pp. 201-208, 1992.

[20] A. Goberman and J. Whitfield, "Acoustics of Infant Pain Cries: Fundamental Frequency as a Measure of Arousal", Perspectives on Speech Science and Orofacial Disorders, vol. 23, no. 1, p. 18, 2013.

[21] P. Zeskind, "Cross-Cultural Differences in Maternal Perceptions of Cries of Low- and High-Risk Infants", Child Development, vol. 54, no. 5, p. 1119, 1983.

[22] Y. Shinya, M. Kawai, F. Niwa, and M. MyowaYamakoshi,, "Preterm birth is associated with an increased fundamental frequency of spontaneous crying in human infants at term-equivalent age', Biology letters. http://dx.doi.org/10.1098/rsbl.2014.0350, 2014.

[23] H. Gilbert, M. Robb and Y. Chen, "Formant frequency development: 15 to 36 months", Journal of Voice, vol. 11, no. 3, pp. 260-266, 1997.

[24] C. Thodén and K. Michelsson, "Sound Spectrographs Cry Analysis in Krabbe's Disease", Developmental Medicine \& Child Neurology, vol. 21, no. 3, pp. 400-402, 2008.

[25] A. Cacace, M. Robb, J. Saxman, H. Risemberg and P. Koltai, "Acoustic features of normal-hearing pre-term infant cry", International Journal of Pediatric Otorhinolaryngology, vol. 33, no. 3, pp. 213-224, 1995.

[26] Y. Kheddache and C. Tadj, "Characterization of Pathologic Cries of Newborns Based on Fundamental Frequency Estimation", Engineering, vol. 05, no. 10, pp. 272-276, 2013.

\section{Publish your research article in AIJR journals-}

$\checkmark \quad$ Online Submission and Tracking

$\checkmark \quad$ Peer-Reviewed

$\checkmark \quad$ Rapid decision

$\checkmark \quad$ Immediate Publication after acceptance

$\checkmark \quad$ Articles freely available online

$\checkmark \quad$ Retain full copyright of your article.

Submit your article at journals.aijr.in

\section{Publish your books with AIJR publisher-}

$\checkmark \quad$ Publish with ISBN and DOI.

$\checkmark$ Publish Thesis/Dissertation as Monograph.

$\checkmark$ Publish Book Monograph.

$\checkmark$ Publish Edited Volume/ Book.

$\checkmark$ Publish Conference Proceedings

$\checkmark \quad$ Retain full copyright of your books.

Submit your manuscript at books.aijr.org 